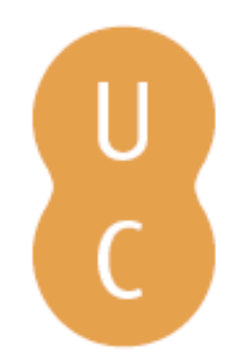

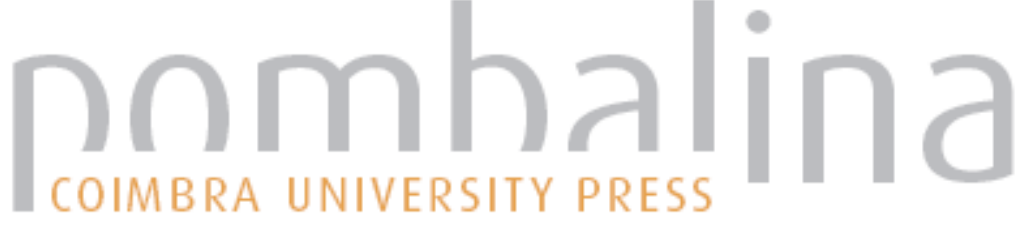

\section{Avaliação de critérios epidemiológicos na população portuguesa}

Autor(es): $\quad$ Pereira, Cristina S.; Soares, Carla G.; Pereira, J. V. Silva

Publicado por: Imprensa da Universidade de Coimbra; RISCOS - Associação

URL

persistente: URI:http://hdl.handle.net/10316.2/34894

DOI: $\quad$ DOI:http://dx.doi.org/10.14195/978-989-96253-3-4_93

Accessed : $\quad$ 26-Apr-2023 11:34:42

A navegação consulta e descarregamento dos títulos inseridos nas Bibliotecas Digitais UC Digitalis, UC Pombalina e UC Impactum, pressupõem a aceitação plena e sem reservas dos Termos e Condições de Uso destas Bibliotecas Digitais, disponíveis em https://digitalis.uc.pt/pt-pt/termos.

Conforme exposto nos referidos Termos e Condições de Uso, o descarregamento de títulos de acesso restrito requer uma licença válida de autorização devendo o utilizador aceder ao(s) documento(s) a partir de um endereço de IP da instituição detentora da supramencionada licença.

Ao utilizador é apenas permitido o descarregamento para uso pessoal, pelo que o emprego do(s) título(s) descarregado(s) para outro fim, designadamente comercial, carece de autorização do respetivo autor ou editor da obra.

Na medida em que todas as obras da UC Digitalis se encontram protegidas pelo Código do Direito de Autor e Direitos Conexos e demais legislação aplicável, toda a cópia, parcial ou total, deste documento, nos casos em que é legalmente admitida, deverá conter ou fazer-se acompanhar por este aviso.

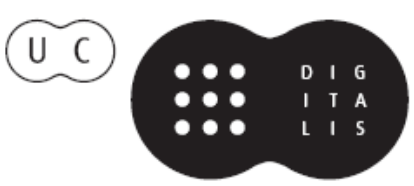



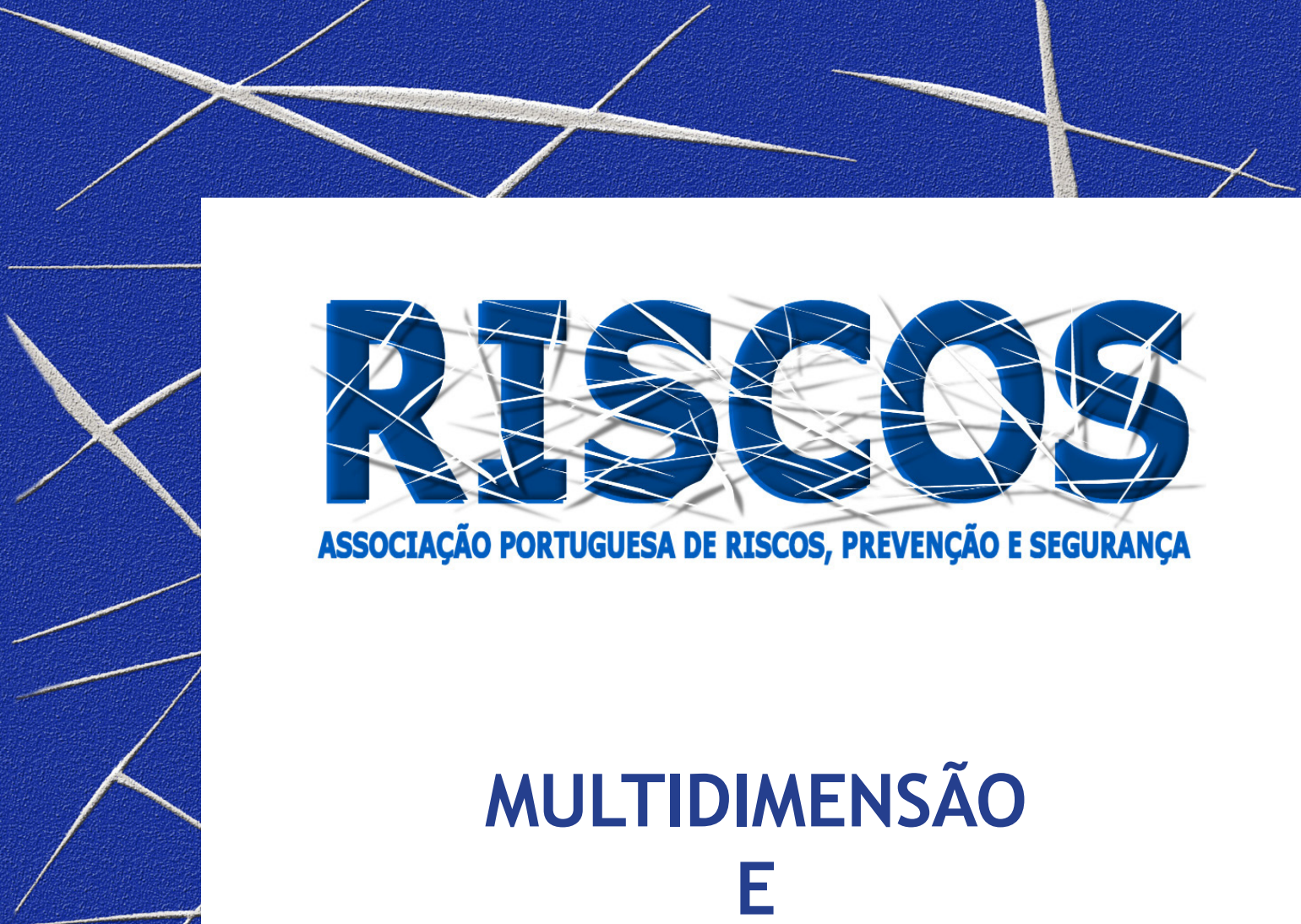

ASSOCIAÇÃO PORTUGUESA DE RISCOS, PREVENCCÃO E SEGURANÇA

MULTIDIMENSÃO

E
TERRITÓRIOS DE RISCO

III Congresso Internacional

I Simpósio Ibero-Americano

VIII Encontro Nacional de Riscos

Guimarães

2014 


\title{
AVALIAÇÃO DE CRITÉRIOS EPIDEMIOLÓGICOS NA POPULAÇÃO PORTUGUESA
}

\author{
Cristina. S. Pereira \\ CHUC- Centro Hospitalar Universitário de Coimbra \\ crisilvapereira@gmail.com \\ Carla G.Soares \\ IDEALMED-Unidade Hospitalar de Coimbra \\ carla.soares@idealmed.pt \\ J. V. Silva Pereira \\ Instituto Superior de Ciências da Informação e da Administração (ISCIA) \\ jvsilvapereira@sapo.pt
}

\begin{abstract}
RESUMO
Há marcadores epidemiológicos utilizados na saúde pública dos países, cuja importância e relevância dependem de inúmeros fatores como localização regional, natalidade, mortalidade e ambiente. Alguns são tão importantes como a natalidade e imigração que alteram a estrutura social e económica. Estudos têm sido feitos para demonstrar sua importância e influência. Em Portugal, o responsável pela avaliação e controlo é o Instituto Nacional de Estatística e a Direção Geral de Saúde. Para que um marcador seja importante numa população ele deverá ser avaliado em diversos âmbitos e ter um significado estatístico relevante como é a imigração na população portuguesa (TABI, INE).Mundialmente, marcadores estudados por metaanálise tem sido considerados significativos e uniformes por toda a bibliografia avaliada(TABII) Concluímos que as técnicas de avaliação de marcadores epidemiológicos utilizadas no nosso pais são compatíveis e devem ser aplicadas de forma crítica.
\end{abstract}

Palavras-chaves: Vigilância epidemiológica, Marcadores populacionais, Saúde pública.

\section{Introdução}

O sistema de vigilância epidemiológica tem por atribuição principal oferecer apoio aos diversos sistemas de controlo nas diferentes populações a nível nacional e internacional. Têm sido implementados por esse organismo normas para avaliação de vários aspetos populacionais através dos marcadores laboratoriais em lato senso como pela identificação de marcadores virais, bacteriológicos, farmacológicos, bem como psicossociais, económicos e culturais.

Todos eles são importantes, mas em cada população a magnitude é diferente devido a inúmeros fatores tais como, localização regional, número da população, natalidade, mortalidade e factores ambientaisAlguns são tão importantes como a natalidade e imigração que modificam totalmente a estrutura social, económica e outros marcadores. ( Ahluwalia IB,2000 ; Fonseca JF.)

Inúmeros trabalhos e livros têm sido escritos para demonstrar sua importância, influência e associações.Na população portuguesa o órgão responsável por esta avaliação é o Instituto Nacional de Estatística, sendo que a Direcção Geral de Saúde faz também o seu controlo.

Os laboratórios deste sistema têm também por atribuição o desenvolvimento tecnológico aplicado à produção de antígenos e anticorpos, assim como o desenvolvimento e padronização de técnicas para diagnóstico e atividades voltadas a oferecer soluções em saúde pública. Praticamente, toda a tecnologia desenvolvida é utilizada pelos laboratórios públicos e privados do sistema nacional de saúde. Estes laboratórios somente executam exames para diagnóstico em situações especiais e mediante solicitação específica e justificável. 
Para que um marcador seja importante numa população deverá ser avaliado de diversos ãngulos e ter um significado estatístico relevante na população estudada, como é a imigração na população portuguesa como se observa na TAB.I do INE(Portal do Instituto Nacional de Estastística)

Em termos metodológicos podem ser utilizados vários métodos de avaliação, tal como revisões bibliográficas dos critérios de avaliação do sistema epidemiológico extraídas de publicações periódicas, jornais e revistas; bem como bases de dados nacionais e internacionais. Foram considerados nesta metodologia todos os objetivos, obedecendo a diferentes versões aplicáveis, adequados ao devido sistema de saúde e realizado a análise da informação obtida em comparação ao marcador estudado.

Em todo o mundo diversos marcadores estudados tem sido adequadamente considerados como mostra a metaánalise realizada por nós, TAB II, com odds ratio significativo e uniforme por quase toda a bibliografia avaliada.

TAB.I- Marcadores epidemiológicos 2000-2007 em Portugal.(INE)

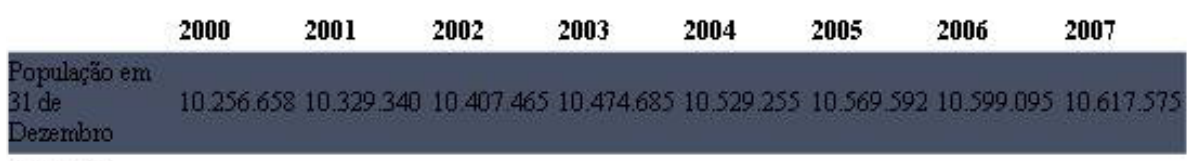

Razão de

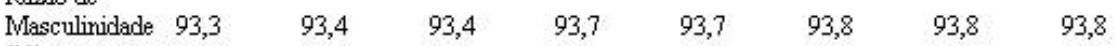

(\%)

\begin{tabular}{|c|c|c|c|c|c|c|c|c|}
\hline $\begin{array}{l}\text { Total de Nados } \\
\text { Viros }\end{array}$ & 120.008 & 112774 & 114.383 & 112515 & 109.262 & 109.399 & 105.351 & 102.492 \\
\hline Total de Óbitos & 105.364 & 105.092 & 106.258 & 108.795 & 101.932 & 107.462 & 101.948 & 103.512 \\
\hline Saldo Natural & 14.644 & 7.682 & 8.125 & 3.720 & 7.330 & $1: 937$ & 3.403 & -1.020 \\
\hline $\begin{array}{l}\text { Saldo } \\
\text { migratónio }\end{array}$ & 47.000 & 65.000 & 70.000 & 63.500 & 47.240 & 38.400 & 26.100 & 19.500 \\
\hline $\begin{array}{l}\text { Vanação } \\
\text { populacional }\end{array}$ & 61.644 & 72.682 & 78.125 & 67220 & 54.570 & 40,337 & 29.503 & 18.480 \\
\hline $\begin{array}{l}\text { Crescimento } \\
\text { Natural } \\
(\%)\end{array}$ & 0,14 & 0,07 & 0,08 & 0,04 & 0,07 & 0,02 & 0,03 & $-0,01$ \\
\hline $\begin{array}{l}\text { Crescimento } \\
\text { Mligratóno } \\
(\%)\end{array}$ & 0,46 & 0,63 & 0,68 & 0,61 & 0,45 & 0,36 & 0,25 & 0,18 \\
\hline $\begin{array}{l}\text { Crescimento } \\
\text { Efectivo } \\
(\%)\end{array}$ & 0,60 & 0,71 & 0,75 & 0,64 & 0,52 & 0,38 & 0,28 & 0,17 \\
\hline
\end{tabular}

(\%)

Fonte: Instituto Nacional de Estatística. Estimativas provisónas de população residente, 2007. Portugal, NUTS II, NUTS III e municípios. Lisboa: INE-IP, 2008 
TAB.II - Resultado da metaanálise realizada com odds ratio $p \geq 1$.

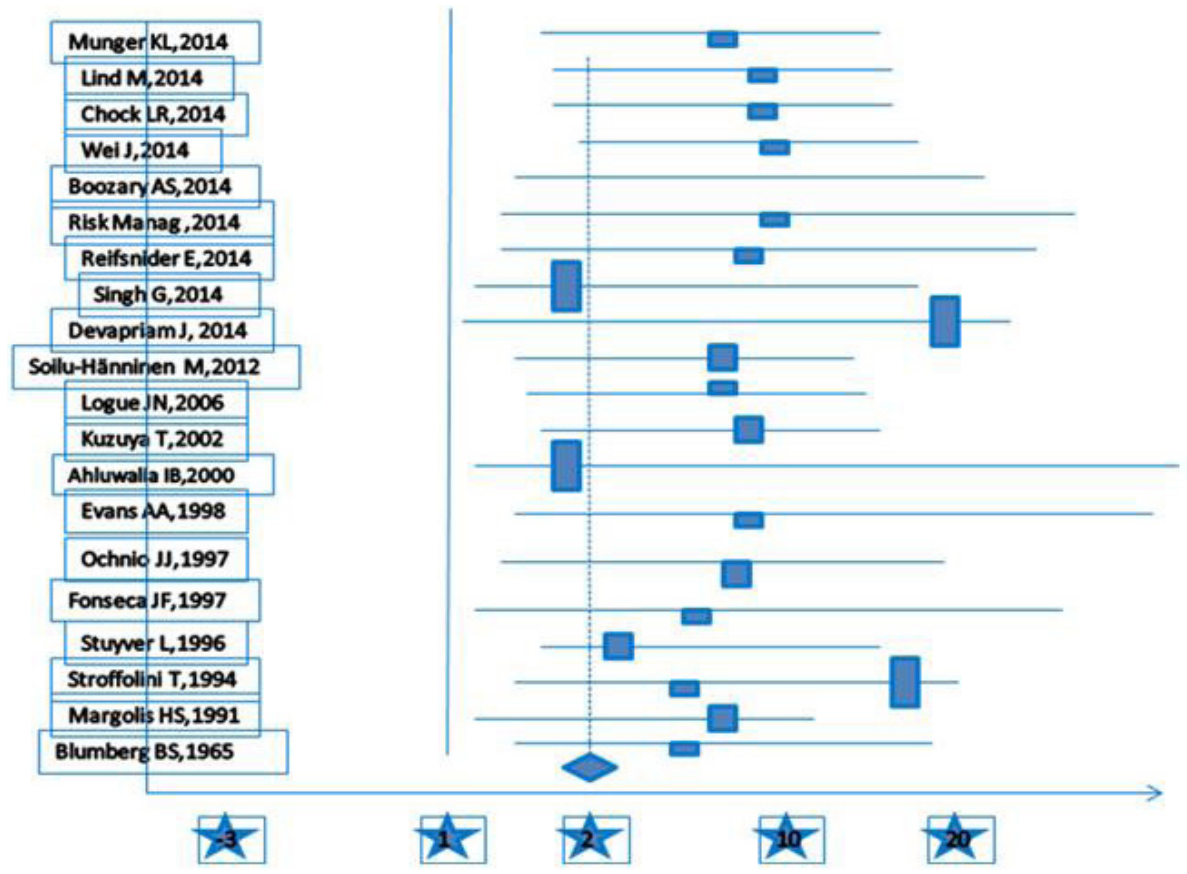

Este trabalho visa avaliar a importância de marcadores epidemiológicos para a determinação de perigos na saúde.( Risk Manag Healthc Policy,2014)

\section{Material e Métodos}

Foram utilizados revisões bibliográficas de marcadores epidemiológicos em vários países através de metaánalise e observada a sua importância estatística quando comparada com dados obtidos em Portugal. A Fig. 1 mostra a sequência da análise dos dados considerada significativa devido ao valor de odds ratio estar acima de 1 .

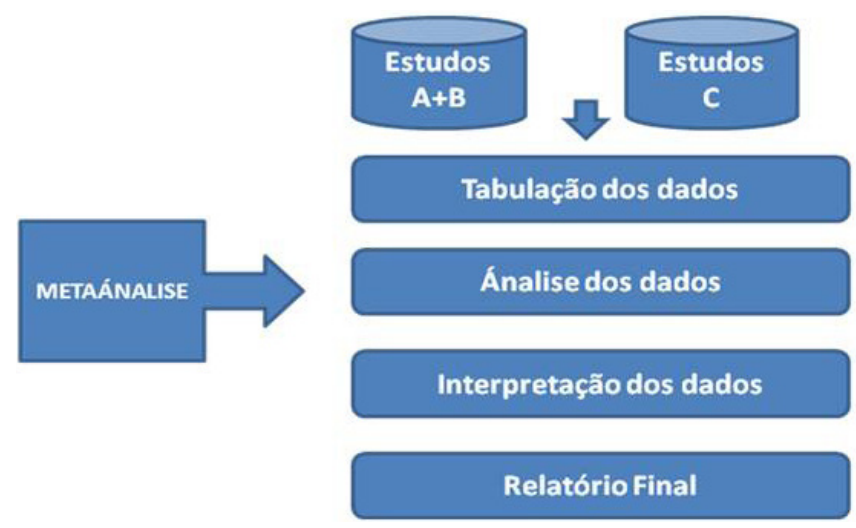

Fig.1-Sequência de análise dos dados realizada. 


\section{Conclusões}

$\mathrm{Na}$ metaánalise realizada, observamos uma distribuição homogénea nos artigos avaliados com odds ratio acima de 1 , na maioria dos estudos utilizados. Isto indica que houve uma escolha adequada na análise, bem como os marcadores aqui assinalados tem um significado relevante nas populações estudadas. Em Portugal, foram utilizados os dados obtidos no INE que apresentam paralelismo evidente. Assim, todas as avaliações dos estudos, leva-nos a crer que têm sido utilizadas metodologias adequadas já que há uma coincidência próxima entre a avaliação dos marcadores populacionais no mundo e em Portugal.

\section{Bibliografia}

Ahluwalia IB, Tessaro I, Grummer-Strawn LM, MacGowan C, Benton-Davis, 2000.S.Georgia's breastfeeding promotion program for low-income women.Pediatrics. Jun;105(6):E85.

Blumberg BS, Alter HJ and Visnich S. A “New” antigen in Leukemia sera. JAMA 1965; 191(7): 541-6.

Boozary AS, Farmer PE, Jha AK. JAMA. 2014 Oct 6. The Ebola Outbreak, Fragile Health Systems, and Quality as a Cure.

Chock LR, Hayes DK, Tomiyasu DW. Insights in public health: the special supplemental nutrition program for women, infants and children: strengthening families for 40 years. Hawaii J Med Public Health. 2014 Sep;73(9):295-300.

Devapriam J, Gangadharan S, Pither J, Critchfield M. Delayed discharge from intellectual disability in-patient units. Psychiatr Bull (2014).

Evans AA, London WT. Epidemiology of hepatitis B. In: Zuckerman Viral hepatitis: Scientific basis and clinical management. 2nd ed. London: Churchill Livingstone;1998. p.107-14.

Fonseca JF. Hepatite D. In: Focaccia R, editor. Hepatites virais. São Paulo: Atheneu; 1997.p.67-9.

Kuzuya T., Nakagawa S., Satoh J., Kanazawa Y., Iwamoto Y., Kobayashi M., Nanjo K., Sasaki A., Seino Y., Ito C, Shima K., Nonaka K., Kadowaki T.; Committee of the Japan Diabetes Society on the diagnostic criteria of diabetes mellitus. Report of the Committee on the classification and diagnostic criteria of diabetes mellitus. Diabetes Res Clin Pract. 2002 Jan;55(1):65-85.

Lind M., Tuomilehto J., Uusitupa M., Nerman O., Eriksson J., Ilanne-Parikka P., Keinänen-Kiukaanniemi S., Peltonen M., Pivodic A., Lindström J. The Association between HbA1c, Fasting Glucose, 1-Hour Glucose and 2-Hour Glucose during an Oral Glucose Tolerance Test and Cardiovascular Disease in Individuals with Elevated Risk for Diabetes. PLoS One. 2014 Oct 6;9(10).

Logue JN. The public health response to disasters in the 21st century: reflections on Hurricane Katrina.J Environ Health. 2006 Sep;69(2):9-13, 26.

Margolis HS, Alter MJ, Hadler SC. Hepatitis B: envolving epidemiology and implications for control. Sem Liver Dis 1991; 2: 84-92. HEPATITES.

Munger KL, Köchert K, Simon KC, Kappos L, Polman CH, Freedman MS, Hartung HP, Miller DH, Montalbán X, Edan G, Sandbrink R, Pohl C. Molecular mechanism underlying the impact of vitamin $D$ on disease activity of MS. Ann Clin Transl Neurol. 2014

Ochnio JJ, Scheifele DW, Ho M. Hepatitis A virus infection in urban children - are preventive opportunities being missed? J Infect Dis 1997; 176: 1610-3.

Portal do Instituto Nacional de Estastistica -www.ine.pt

Reifsnider E1, Bishop SL, An K, Mendias E, Welker-Hood K, Moramarco MW, Davila YR. We Stop for No Storm: Coping with an Environmental Disaster and Public Health Research. Public Health Nurs. 2014 Oct 5. 
Risk Manag Healthc Policy. 2014 Sep 24;7:163-75. Evidence from the national health account: the case of Dubai.

Singh G, Helman D, Brady SK. American College of Physicians, Hawai'i Chapter, Annual Meeting 2014. Hawaii J Med Public Health. 2014 Sep;73(9 Suppl 1):2.

Soilu-Hänninen M1, Aivo J, Lindström BM, Elovaara I, Sumelahti ML, Färkkilä M, Tienari P, Atula S, Sarasoja T, Herrala L, Keskinarkaus I, Kruger J, Kallio T, Rocca MA, Filippi M. A randomised, double blind, placebo controlled trial with vitamin D3 as an add on treatment to interferon $\mathrm{B}-1 \mathrm{~b}$ in patients with multiple sclerosis. J Neurol Neurosurg Psychiatry. 2012 May;83(5):565-71.

Stroffolini T, Cialdea L, Catapano R, Palumbo F, Novaco F et al. Incidence and risk factors of acute Delta hepatitis in Italy: Results from a national surveillance system. J Hepatol 1994; 21: 1123-6.

Stuyver L. Genótipos de VHC e métodos de genotipagem. News Lab 1996; 15: 147-52.

Wei J1, Hansen A, Zhang Y, Li H, Liu Q, Sun Y, Xue S, Zhao S, Bi P. PLoS One. 2014 Oct 6;9(10). The Impact of Climate Change on Infectious Disease Transmission: Perceptions of CDC Health Professionals in Shanxi Province, China. 